\title{
The Role of Propagation Effects in Airborne RF Communication Systems Design
}

\author{
Thomas Morton and Kevin Sickles \\ Air Force Research Laboratory, Sensors Directorate \\ Wright-Patterson AFB, OH. \\ thomas.morton@wpafb.af.mil and kevin.sickles@wpafb.af.mil
}

\begin{abstract}
Recent years have seen the emergence of Unmanned Aerial Systems performing roles that use various Radio Frequency (RF) systems. Thus, there is a need to use accurate and broadly capable signal propagation models to capture multi-path effects as well as atmospheric effects in system requirements analysis. One such model, the Variable Terrain Electromagnetic Parabolic Equation (VTRPE) model, enables the required analysis for a wide range of applications. The work presented in this paper uses VTRPE to analyze signal propagation in multi-path environments and places the results in a system design context drawing conclusions to aid in airborne RF systems design.
\end{abstract}

\section{INTRODUCTION}

Airborne Radio Frequency (RF) systems are generally comprised of some combination of antenna, receiver, transmitter, and processing subsystems, and have numerous military applications. These include radar, communication radio relay, and signal collection among many others. The design of a given airborne RF system depends on system functions and the operational environment.

The emergence of Unmanned Aerial Systems (UAS) has naturally led to proposals for fitting them with RF payloads. Air-to-ground and ground-to-air functions are an important subset of RF applications proposed for UAS. Unlike air-to-air functions, where the number of multi-path signal returns is small, the air-to-ground and ground-to-air functions are susceptible to multi-path return. Multi-path signals arrive at the airborne receiver delayed in time with respect to the direct path signals. The resulting phase differences cause constructive or destructive interference patterns. Moreover, since the aircraft is moving, the interference patterns vary cyclically [1]. The ground or water, acting as the multi-path source, is characterized by its elevation, surface roughness, and dielectric properties, all of which contribute to the signal's propagation characteristics.

The Navy, traditionally more concerned than the Air Force with multi-path propagation and anomalous atmospheric conditions, has been developing advanced propagation models for years [2]. The literature is mature with reports, measurements, and analysis regarding coastal and open sea propagation including ducting and sea clutter [3]-[8]. With the recent advent of UAS, large and small, high altitude and low altitude, Air Force designers must now consider signal propagation in many of their RF system designs. While there are a number of papers proposing and outlining the use of UAS as communication relay links [9]-[13], there are few papers analyzing the propagation effects of terrain environments on the UAS communication link [14], [15].

There are a number of empirically derived multi-path and atmospheric fading models that can be used to analyze the terrain environment [16]-[19]; however, most of these models are best suited to specific conditions and require significant effort and expertise from the user.

This paper demonstrates the importance of including signal propagation effects for airborne RF functions. The particular case considered here is communication link, but the analysis can be extended to other functions such as radar. It considers sea and land environments as well as ducting and standard atmospheres. For the land environment, both ground-to-air and air-to-ground communication links are considered. Calculated figures of merit include received power and signal to noise ratio. The latter is discussed in terms of bit error probability and communication link margin. Most of the parameters in the presented communication link scenarios are given assumed values with the exception of the propagation factor which is modeled by the Navy developed VTRPE (Variable Terrain Electromagnetic Parabolic Equation) code. VTRPE provides a simple user interface and is broadly capable of handling most scenarios. The modeled results show significant effects on the figures of merit resulting from the terrain and ducting conditions.

The remainder of the paper is organized as follows. The problem formulation discusses the communication link trade space and the propagation factor. The results section follows and presents communication link figures of merit for two modeled scenarios: sea-based ducting and standard atmospheric conditions and land-based propagation in rough, mountainous terrain. The results are followed by a conclusion and list of references. 


\section{FORMULATION OF THE PROBLEM}

Transmitting and receiving timely, useful information in a military setting can range from small unit tactics to large scale integrated networks. Small or large, the problem is best characterized as a systems engineering challenge that brings many diverse fields of expertise together.

\section{A. Communication Link}

Let us consider a simple one-way communication link as depicted in Fig. 1. A transmitting system radiates electromagnetic energy in the form of a Radio Frequency (RF) signal. The signal propagates through the environment where it is captured by the receiving antenna which routes it to the receiver for detection and processing. The transmitting and receiving systems may be stationary or mobile, ground-based, ship-based, space-based, or airborne.

The information exchange requirement, or bit rate, ranges from 10's of kbs (kilobits per second) to Gbps (billions of bits per second), and the designs may involve constraints such as limited space and payload for an Unmanned Aerial System (UAS) or limited power for a space-based system. Hardware and processing designs evolve with technology. This evolution helps the designer. The environment, on the other hand, must be characterized and understood. Any given environment's effect on signal propagation, moreover, is subject to change with weather, season, and time of day. There are concepts such as cognitive radio [20] that seek to optimize performance in response to a changing environment but more fundamentally the challenge is to understand and characterize the environment. Before discussing the propagation environment let us define the transmit and receive systems in greater detail and develop the necessary equations for analysis.

The key system performance measure is signal to noise ratio, or the ratio of power available at the receiver antenna output terminals, $P_{r}$, to the thermal noise in the receiver, $N$ :

$$
\frac{P_{r}}{N}=P_{t} G_{t}\left(G_{r} / N\right) F^{2} L_{r x} L_{t x} L_{f}
$$

where $P_{t}$ is the power available to the transmit antenna, $G_{t}$ is the peak gain of the transmit antenna, and $G_{r}$ is the peak gain of the receive antenna. The receive and transmit system loss terms, $L_{r x}$ and $L_{t x}$, can include a number of factors such as impedance mismatch between transmission lines and antennas, polarization mismatch, and attenuation losses. The free-space propagation loss, $L_{f}=[\lambda /(4 \pi R)]^{2}$, accounts for power density loss caused by spherical waveform spreading with range; note its one-way dependence on $\mathrm{RF}$ wavelength, $\lambda$, and Range, R. The propagation factor, $F$, central to the topic of this paper, is discussed in the next section.

A common formulation for (1) used for digital links is given by

$$
\frac{P_{r}}{N_{0}}=P_{t} G_{t}\left(G_{r} /(T k)\right) F^{2} L_{r x} L_{t x} L_{f}
$$

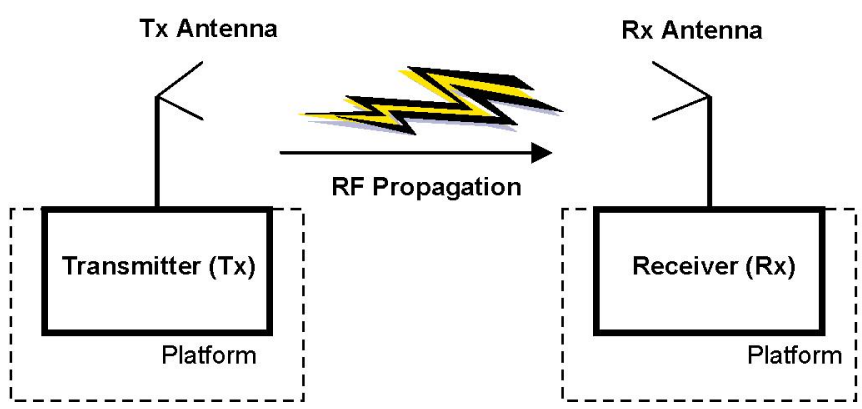

Figure 1. One-way communication link.

where $N$ is replaced by the ratio of the noise spectral density to the product of Boltzmann's constant, $\mathrm{k}$, and the effective system noise temperature, $N=N_{0}(k T)$. The effective system noise temperature, $T$, is a composite of noise contributions and depends on where the antenna is 'looking' as well as the noise performance of the receiver [21].

To quantify the information rate of the communication link, (1) may be expressed as

$$
\frac{P_{r}}{N_{0}}=\frac{E_{b}}{N_{0}} S
$$

where $E_{b} / N_{0}$ is the bit energy per noise spectral density and $S$ is the bit rate in bits per second (bps). Substituting (3) into (2) gives a form well suited for quantifying bit error rate:

$$
\frac{E_{b}}{N_{0}}=P_{t} G_{t}\left(G_{r} /(T k)\right) F^{2} L_{r x} L_{t x} L_{f} S .
$$

Equations (1)-(4) encapsulate the trade-space for the oneway communication link system design.

\section{B. Propagation Factor}

The propagation factor, $F$, in (1), (2) and (4), accounts for the situation where the receiver may not be in the mainlobe of the transmit antenna, and accounts for non free-space propagation. Non free-space propagation includes multi-path interference, terrain dielectric properties and roughness, sea dielectric properties and sea states, and atmospheric effects: refraction, diffraction, attenuation, and absorption. The International Telecommunications Union (ITU) is a good source for information about RF signal propagation and for analytical models to characterize propagation. Many propagation models, both analytical and numerical, exist. The results in this paper are generated with a Navy developed propagation model named VTRPE (Variable Terrain Radiowave Parabolic Equation) [24]. VTRPE models all of the non free-space propagation effects listed above and provides the propagation factor as its primary output. We mention in passing that validation of VTRPE is documented, at a minimum, in [ 7], [22 ], [23 ].

The results section of this paper presents results for which standard atmospheric and ducting atmospheric conditions hold. Thus, a few preliminary words about these atmospheric conditions are appropriate. Atmospheric ducts usually occur in coastal or ocean regions, can exist at the surface or as an 
elevated layer, and for certain RF frequencies act to trap the RF waves by refracting, or bending them, towards the earth's surface. In radar systems such bending can result in dramatic increases in detection ranges or in anomalous detections. Several phenomena, including temperature inversions, cause ducting; in fact, night-time cooling of deserts has been reported to cause temperature-inversion surface ducts over desert areas.

Fig. 2 shows atmospheric profiles for standard and ducting conditions. These results are taken from [4]: for two springsummertime periods (2002-03) researchers collected and analyzed atmospheric radiosonde data for the Cheju, Korea region. Using statistical techniques they characterized most probable duct, non-duct and standard atmospheric refractivity profiles. Ducts are indicated by a negative slope in the modified refractivity profile vs. height.

Note that ducting is only one of several anomalous atmospheric conditions. Others, not discussed here, include subrefraction and superrefraction.

\section{RESULTS}

The results presented in this section are meant to demonstrate the importance of including RF propagation effects in communication link trade-space analysis. Two nominal scenarios are presented to quantify the demonstration. VTRPE provides the propagation factor; we assign 'reasonable' values to all other parameters. Each of these other parameters may in general have values determined by their own modeling tools and careful analysis in the same way that VTRPE is providing the propagation factor. Indeed, it is just such a larger integrated systems modeling effort that we are working towards

The first scenario illustrates the effect of atmospheric surface ducting on received signal power over a coastal region. The second scenario illustrates ground-to-air and airto-ground signal to noise ratios for rugged, mountainous terrain.

\section{A. Scenario $1-$ Cheju}

As discussed in the previous section and shown in Fig. 2 we have access through [4] to statistically derived surface duct profiles for the Cheju, Korea area. Fig. 3 shows the location of Cheju, an island off the southern coast of Korea.

In [4] the authors give an example in which the received power, $P_{r}$, is the figure of merit. The communication is for two ships where the transmit and receiving antennas are at $30 \mathrm{~m}$ above the surface. Removing the receiver noise from (1) and assuming no receiver or transmitter system losses gives

$$
P_{r}=P_{t} G_{t} G_{r} L_{f} F^{2}
$$

where $P_{t}=10 \mathrm{w}, G_{t}=20 \mathrm{~dB}, G_{r}=20 \mathrm{~dB}$, and $\lambda=0.0638 \mathrm{~m}$ (frequency $=4.7 \mathrm{GHz}$ ). The atmospheric profiles from Fig. 2 are fed into VTRPE and VTRPE generates the propagation factor results shown in Fig. 4. Fig. 4 is a composite of three separate VTRPE runs stacked vertically. To insure adequate display space the upper altitudes above the duct are cut off. In

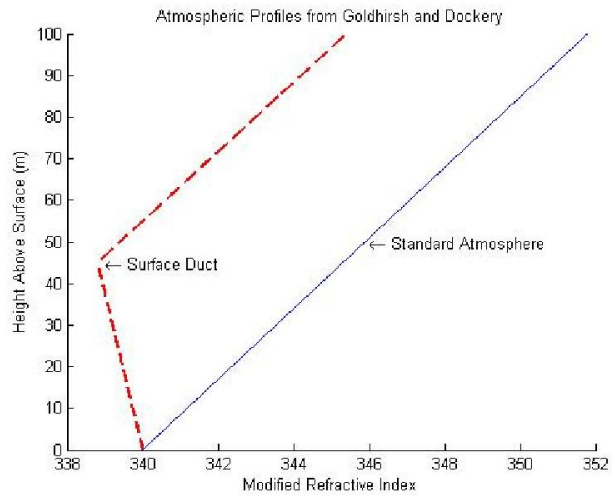

Figure. 2. Atmospheric profiles for surface duct and standard atmosphere

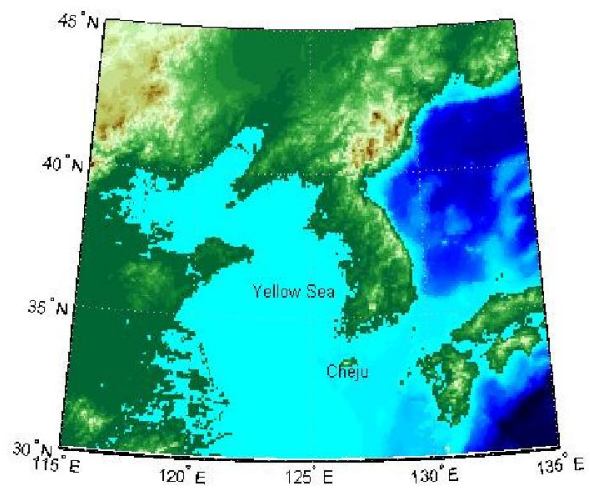

Figure 3. Cheju, Korea and surrounding region.

each case the transmitter is located at the far left of the plot where the range profile begins. The model then propagates the wave only in the direction of increasing range and only in the plane of the range profile. The range profile is zero altitude mean sea level in this case. The graphic shows the propagation factor as color coded data placed on an altitude (m) vs. range grid $(\mathrm{km})$. In Fig. 4 the top plot is for the most probable duct condition and the middle plot is for the standard atmosphere condition. The bottom plot is also for the most probable duct condition; however, here the frequency is 300 MHz so that the resulting $1 \mathrm{~m}$ wavelength is now too long to be trapped by the duct. As an aside, note that the strength of a duct is quoted in terms of the maximum wavelength trapped by the duct. The exact expression is given in [5].

Fig. 4 clearly shows the characteristic constructive and destructive lobing at the closer ranges, particularly for the higher frequency. In the top plot the duct is clearly visible at all ranges for altitudes up to about $45 \mathrm{~m}$ where the surface duct ends as indicated by Fig. 2. At $300 \mathrm{MHz}$ the duct has no effect on the propagation factor.

Fig. 5 plots received power vs. range for the most probable duct and standard atmosphere using the VTRPE propagation factor and the other parameter values given above. The frequency is $4.7 \mathrm{GHz}$ for all three line plots. Note how the ducting condition results in increased received power 


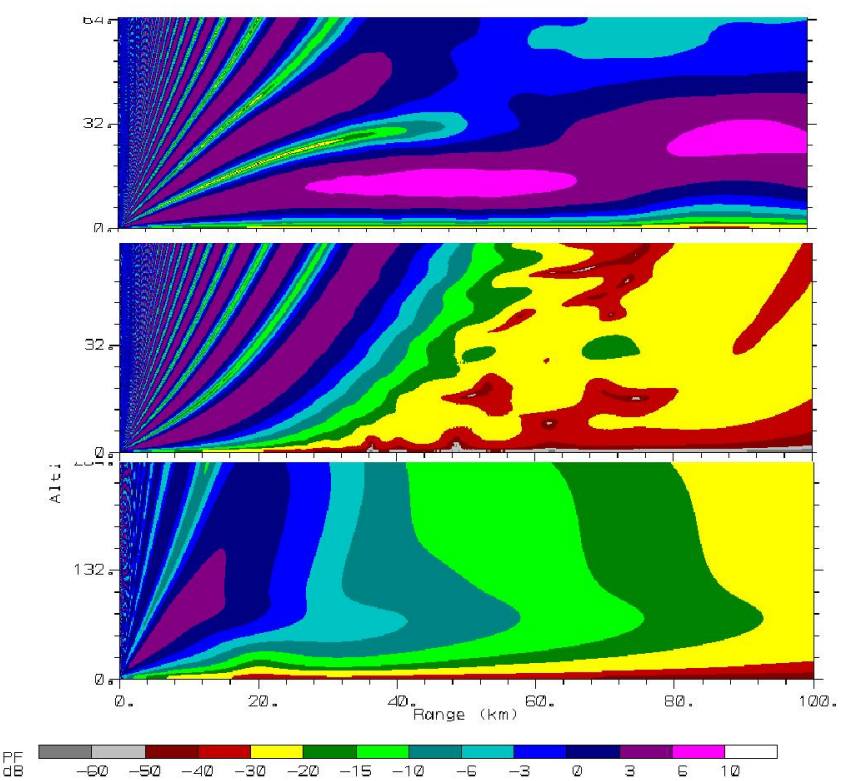

Figure 4. Top plot: surface duct (4.7 GHz); Middle plot: standard atmosphere (4.7 GHz); Bottom plot: surface duct (300 MHz).

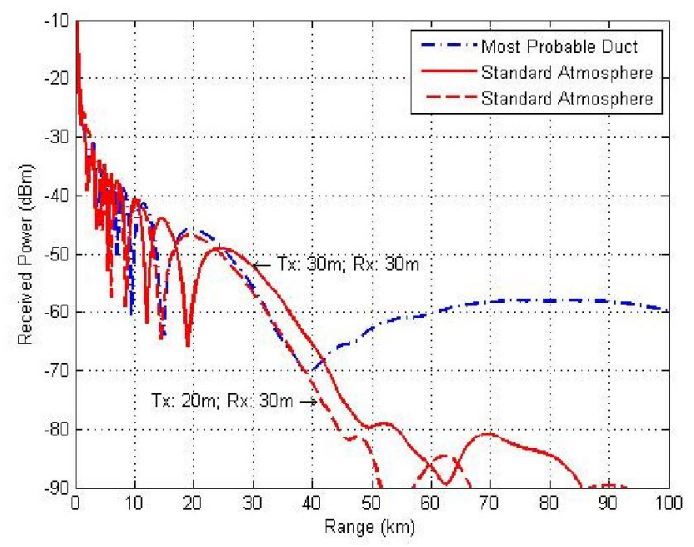

Figure 5. Received power vs. range for surface duct and standard atmosphere.

compared to the standard conditions out to $100 \mathrm{~km}$. Two standard atmosphere plots are shown, one with the receiver at $30 \mathrm{~m}$ and one with it at $20 \mathrm{~m}$. These results track closely to those given in [4] where the propagation factor is modeled using a tool called TEMPER (Tropospheric Electromagnetic Parabolic Routine).

\section{B. Scenario 2 - Bosnia}

Our second scenario moves from water to land and considers terrain effects on the propagation factor. This scenario models only the standard atmosphere. Fig. 6 shows a mapping of Digital Terrain Elevation Data (DTED-2) taken from a 1 degree latitude by 1 degree longitude cell over Bosnia. The bar scale indicates elevation in meters above Mean Sea Level (MSL). Since VTRPE only accepts terrain profiles along the geodesic between two points on the earth, two such points, marked by the stars at the end of the dotted line, are selected as terminal points for a geodesic profile and

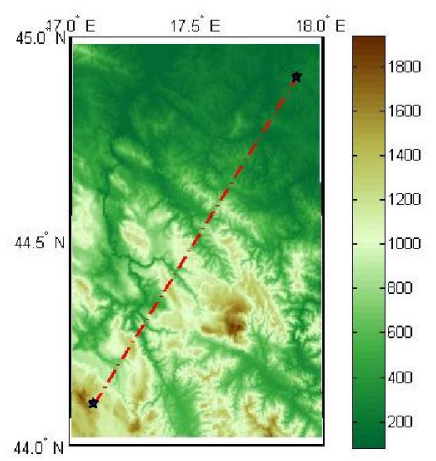

Figure 6. Mapping of Bosnia DTED data with geodesic indicated.

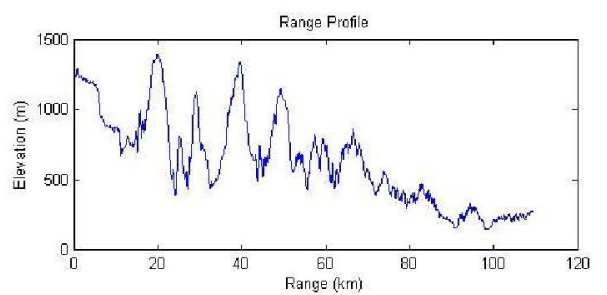

Figure 7. Range profile for the geodesic defined in Fig. 6.

the profile plotted in Fig. 7. Although the terrain is rugged and mountainous, Fig. 7 greatly exaggerates the elevation gradients because the elevation is plotted in meters and the range in kilometers.

The figure of merit for scenario 2 is $\mathrm{E}_{\mathrm{b}} / \mathrm{N}_{0}$ as given by (4). Table 1 lists the parameter values used in the scenario. Recall that in its graphics plots of propagation factor VTRPE always places the transmitter at the beginning of the range profile and propagates the wave only in the direction of increasing range and only in the plane of the profile.

Fig. 8 shows the propagation factor for the case where the transmitter is $3 \mathrm{~m}$ above the ground and the transmit antenna is a monopole with near omni-directional pattern. The shadowing caused by the mountains is most prevalent but there are also widespread areas of constructive interference rising from the ground to the upper altitudes.

The plots shown in Fig. 9 are produced by taking propagation factor results at receiver heights of $3 \mathrm{~m}, 10 \mathrm{~m}$, $1 \mathrm{~km}$, and $5 \mathrm{~km}$. Note that these altitudes are above the ground level directly beneath them so their MSL altitude as a function of range varies in concert with the terrain profile plus a constant offset. Fig. 9a shows the signal to noise ratio vs. range for these altitudes and Fig. $9 \mathrm{~b}$ compares the propagation factor for these altitudes with the free-space propagation loss. Fig. 10, which shows the probability of bit error vs. signal to noise ratio for binary phase shift (BPS) modulation, is included to help interpret Fig. 9a. Fig. 9b shows how the free- 
TABLE 1. System Parameter Values for Scenario 2

\begin{tabular}{|c|c|}
\hline System Parameter & Value \\
\hline $\mathrm{P}_{\mathrm{t}}$ : Transmit Power & $10 \mathrm{dBw}$ \\
\hline $\mathrm{G}_{\mathrm{t}}:$ Tx. Antenna Peak Gain & $5 \mathrm{~dB}$ \\
\hline $\mathrm{G}_{\mathrm{I}}:$ Rx. Antenna Peak Gain & $5 \mathrm{~dB}$ \\
\hline T: System noise temp. & $\mathrm{T}=\mathrm{T}_{\mathrm{a}}+\left(10^{\mathrm{Fdb} / 10}-1\right) 290$ \\
\hline $\begin{array}{l}\mathrm{T}_{\mathrm{a}} \text { : Antenna temp. } \\
\mathrm{Fdb} \text { : Rx. noise figure }\end{array}$ & $=40+\left(10^{1}-1\right) 290=2650 \mathrm{~K}$ \\
\hline K: Boltzmann's constant & $-228.6 \mathrm{dBw} / \mathrm{K}-\mathrm{Hz}$ \\
\hline S: Data rate & $500 \mathrm{kbps}$ \\
\hline R: Range & $0 \mathrm{~km}$ to $110 \mathrm{~km}$ \\
\hline
\end{tabular}

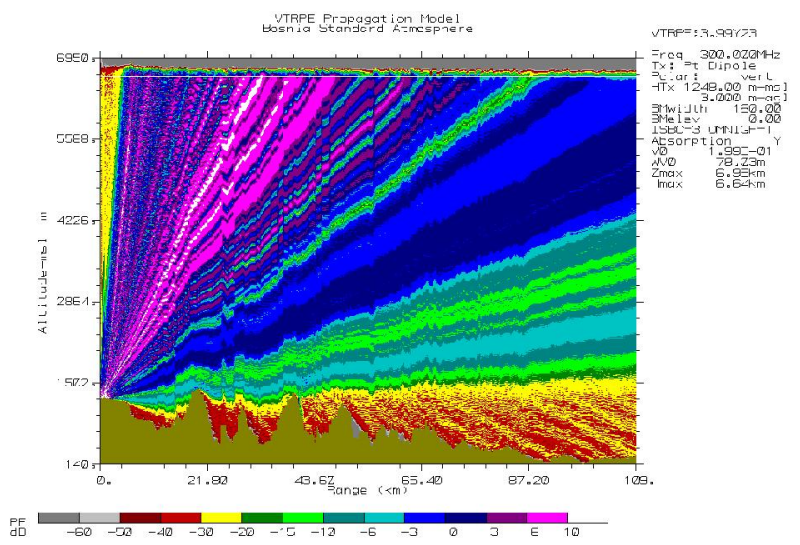

Figure 8. Propagation Factor for ground-based monopole.

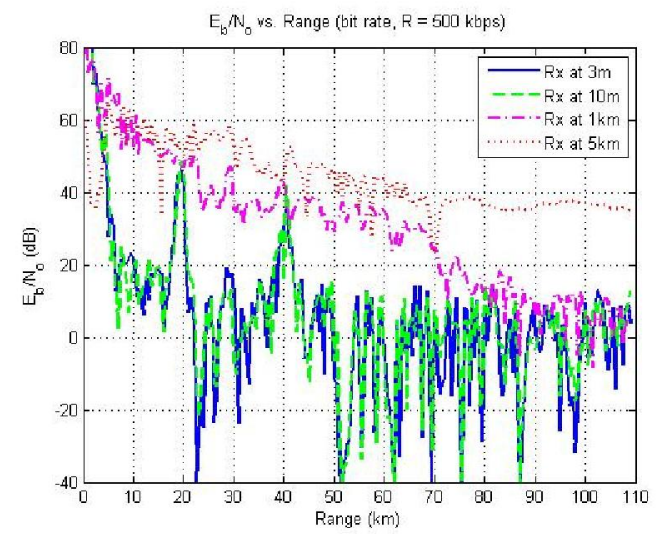

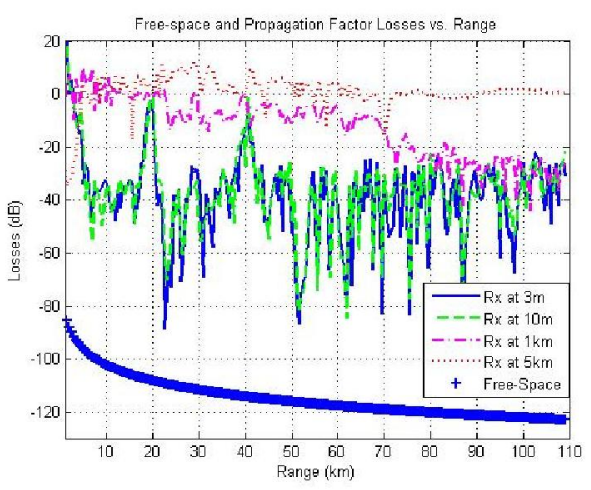

Figure 9a (top): Signal to noise ratio vs. range for four receiver altitudes. Figure 9b (bottom): Comparison of free-space loss to propagation factors for four receiver altitudes.

space loss smoothly descends, predictably losing $6 \mathrm{~dB}$ per octave of range, while the propagation factor jumps wildly in response to the rugged terrain.

As an example, a bit error probability of less than $10^{-4}$ requires a signal to noise ratio of $\mathrm{E}_{\mathrm{b}} / \mathrm{N}_{0} \approx 8.2 \mathrm{~dB}$. Subtracting the required signal to noise ratio of $8.2 \mathrm{~dB}$ from the modeled signal to noise ratio in Fig. 9a gives the link margin shown in Fig. 11. Fig. 11 is a 'stop-light' chart showing regions of marginal (yellow), good (green), and bad (red) link margins and indicates that the receivers at $3 \mathrm{~m}$ and $10 \mathrm{~m}$ fail at ranges beyond $20 \mathrm{~km}$.

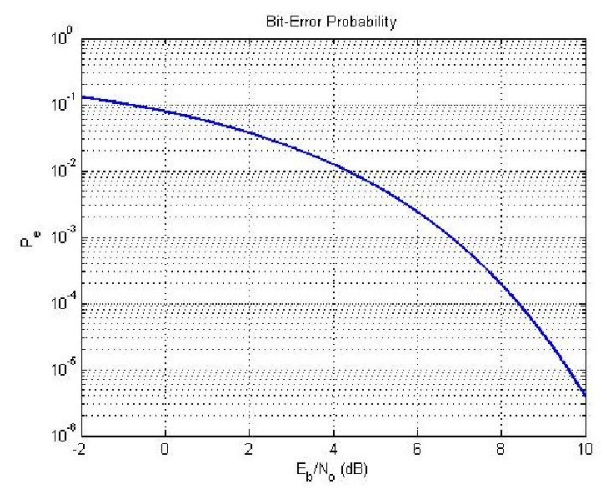

Figure 10. Bit error probability vs. signal to noise ratio for Binary Phase Shift modulation. 


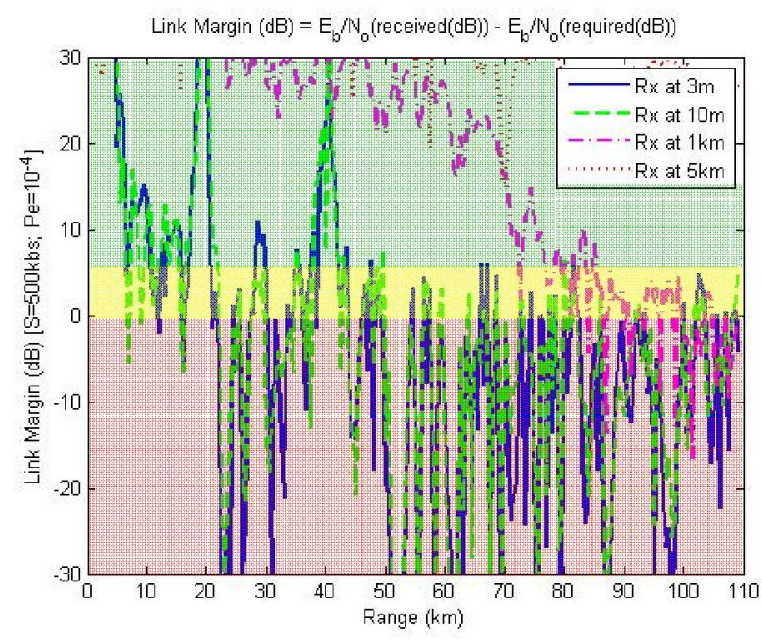

Figure 11. Link margin 'stop-light' chart showing marginal (vellow), good (green), and poor (red) regions of performance for several receiver altitudes.

As a final example let us consider several air-to-ground scenarios with the airborne transmitter located at $1000 \mathrm{~m}$, $3000 \mathrm{~m}$, and $5000 \mathrm{~m}$ above the terrain. The transmit antenna is a monopole with nearly omni-directional pattern. Stacking the propagation outputs vertically and cutting off the upper altitudes results in Fig. 12 where the coverage benefits gained by moving the airborne link up in altitude are indicated by the various mountain shadowings.

Before concluding this section note that in reality the terrain has areas of forestation. As far as the authors can determine, VTRPE does not explicitly model the effect of such diffuse clutter although the model does have inputs for surface roughness.

\section{CONCLUSION}

A simple RF communication link may be characterized by a transmit system, a propagation environment, and a receive system. Radio frequency (RF) propagation in the communication link is quantified by a term called the propagation factor which accounts for non free-space propagation including multi-path interference, terrain and ocean properties, and atmospheric refractivity and absorption. This paper has focused on the propagation environment in the larger context of the communication link problem, although it should be noted that the analysis tools used here are capable of a wider range of RF systems analysis including radar functions. The propagation factor at relatively low altitudes, over ocean and terrain, and for standard and ducting atmospheres has been modeled with a Navy developed code called VTRPE (Variable Terrain Radiowave Parabolic Equation).

For the atmospheric ducting condition over ocean the paper has shown results clearly indicating the presence of ducting and used those results, along with standard atmosphere results, to calculate received signal powers for a postulated C-band communication link. These results show received powers in excess of $20 \mathrm{dBm}$ for the ducting atmosphere compared to the standard atmosphere, and that the

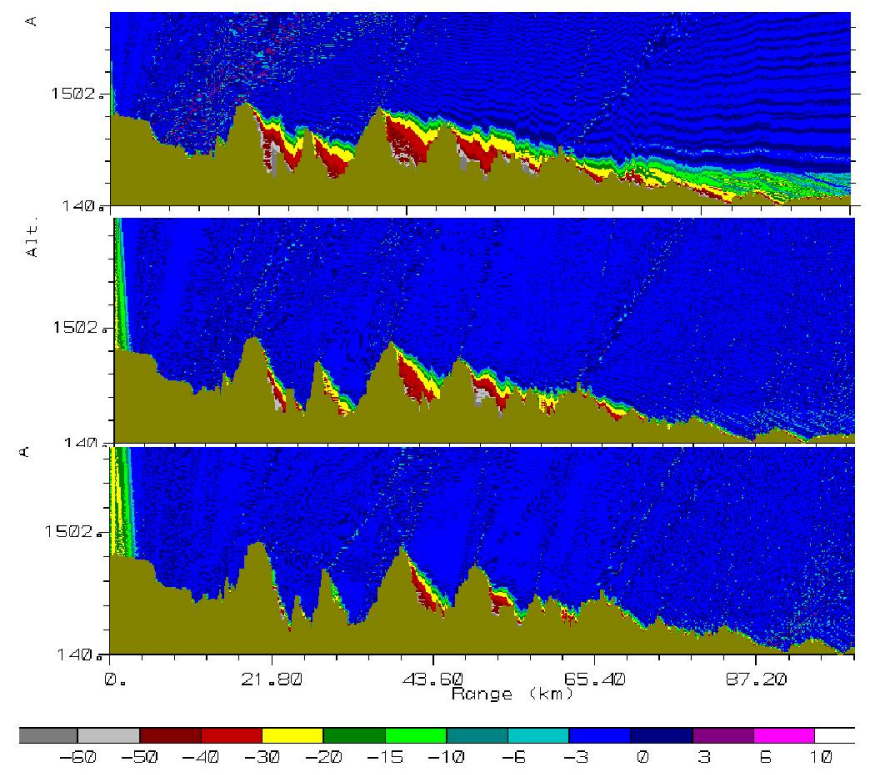

Figure 12. Air-to-ground transmission coverage. Top: altitude of transmitter, $1000 \mathrm{~m}$; middle: altitude of transmitter, $3000 \mathrm{~m}$; bottom: altitude of transmitter, $5000 \mathrm{~m}$.

results track closely to previous results generated with a different propagation model [4].

For the pattern factor over terrain, a range profile of rugged, mountainous land, extracted from Digital Terrain Elevation Data (DTED-2), was input into VTRPE and groundbased and airborne UHF propagation factors generated. Signal to noise ratios for the ground-based system were calculated and related to bit error probabilities. For this specific scenario, the modeled results indicated that for a required bit rate of $500 \mathrm{kbps}$ and bit error probability of less than $10^{-4}$, only airborne receivers would reliably perform at ranges greater than $20 \mathrm{~km}$. The airborne transmitter case demonstrated how area coverage increases with transmitter altitude.

The main contribution of the paper has been to demonstrate the importance of including $\mathrm{RF}$ propagation effects in the communication link trade-space and to demonstrate the utility of using an effective propagation model such as VTRPE as part of the systems engineering effort.

\section{ACKNOWLEDGMENT}

The authors wish to acknowledge Frank J. Ryan of SPAWAR for his generous help in learning some of VTRPE's ins and outs.

\section{REFERENCES}

[1] L.V. Blake, "Prediction of Radar Range," ch. 2 in Radar Handbook, $2^{\text {nd }}$ ed., M. Skolnik ed., McGraw-Hill, 1990.

[2] A.E. Barrios, "Considerations in the development of the advanced propagation models (APM) for U.S. Navy applications," Proceedings of the 2003 International Radar Conference, 3-5 Sept., 2003. 
[3] B. Spaulding, C. Nunn, M. Levitas, "Sea clutter reflectivity in ducted propagation," The Record of the IEEE 2000 Radar Conference, pp. 335-340.

[4] J. Goldhirsh, D. Dockery, "Propagation characteristics for coastal region of South Korea and their impact on communications systems," 2004 IEEE Military Communications Conference, pp. 460-465.

[5] I.M. Brooks, A.K. Goroch, D.P. Rogers, "Observations of strong surface radar ducts over the Persian Gulf," Journal of Applied Meteorology, vol. 38, issue 9, September, 1998, pp. 1293-1310.

[6] W.E. Devereux, J.V. Egmond, "Effects of ducting on radar operation in the Persian Gulf," Proceedings of 1989 National Radar Conference, pp. 174-181.

[7] B. Spaulding, D. Horton, H. Pham, "Wind aspect factor in sea clutter modeling," 2005 IEEE International Radar Conference.

[8] D.G. Money, J. Branson, M. Hooker, A. Mabogunje, "Radar littoral environment predictions and measurements," Radar 97 (Conf. Publ. No. 449), pp. 164-168.

[9] T.H. McKinney, C.T. Johnson, R.B. Mouldin, "Airbone communications node risk reduction strategy," Proceedings 97 MILCOM, 2-5 November, 1997, vol. 1, pp. 398-402.

[10] J.L. Hillman, S.D. Jones, R.A. Nichols, I.J. Wang, "Communication network architectures for the Army Future Combat System and objective force," Proceedings 2002 MILCOM, 7-10 October, 2002, vol. 2, pp. 1417-1421.

[11] I. Rubin, R. Zhang, "Placement of UAVs as communication relays aiding mobile ad hoc wireless networks," Proceedings IEEE Military Communications Conference, 29-31, October, 2007, pp. 1-7.

[12] Maj. F.J. Pinkney, D. Hampel, S. DiPierro, "Unmanned Aerial Vehicle (UAV) communications relay," Proceedings IEEE Military Communications Conference, 1996, 21-24, October, 1996, vol. 1, pp. 47-51.

[13] S. Seidel, T. Krout, L. Stotts, "An adaptive mobile ad-hoc radio backbone system," IEEE Workshop on Networking Technologies for Software Defined Radio Networks, 2006, 25 September, 2006, pp. 1-7.

[14] C. Cerasoli, "An analysis of unmanned airborne vehicle relay coverage in urban environments," Proceedings IEEE Military Communications Conference, 2007, 29-31, October, 2007, pp. 1-7.

[15] T. Wellnitz, O. Nagy, "Wave propagation modeling for airborne communication systems," IEEE Antennas and Propagation Society International Symposium, 20-25, June, 2004, vol. 3, pp. 2235-2238.

[16] H. Goldman, "Mathematical analysis of the three-ray dispersive fading channel model," IEE Proceedings-1, vol. 138, no. 2, April 1991, pp. 8794.

[17] S. Loyka, A. Kouki, F. Gagnon, "Fade depth prediction on wireless microwave links using two-ray multipath model," Canadian Conference on Electrical and Computer Engineering, 2001, vol. 1, pp. 493-498.

[18] O. Sasaki, T. Akiyama, "Multipath delay characteristics on line-of-sight microwave radio system," IEEE Trans. on Communications, vol. Com27, no. 12, December, 1979, pp. 1876-1886.

[19] M. Shafi, "Influence of terrain-induced reflections on the performance of high-capacity digital radio systems," IEEE Trans. on Communications, vol. 36, no. 3, March, 1988, pp. 245-251.

[20] J.F. Hauris, D. He, G. Michel, C. Ozbay, "Cognitive radio and RF communications design optimization using genetic algorithms," IEEE Military Communications Conference, 29-31, October, 2007.

[21] B. Sklar, Digital Communications: Fundamentals and Applications ( $2^{\text {nd }}$ ed.), 2001 Prentice Hall PTR.

[22] Capt. M.K. Doggett, An Atmospheric Sensitivity and Validation Study of the Variable Terrain Radio Parabolic Equation Model (VTRPE). Thesis, March, 1997, Air Force Institute of Technology.

[23] Naval Command, Control and Ocean Surveillance Center, RDT\&E Division, Independent Research 1996 Annual Report, Technical Document 2933, October, 1996.

[24] F.J. Ryan, "User's Guide for the VTRPE (Variable Terrain Radiowave Parabolic Equation) Troposheric/Ionospheric Electromagnetic Propagation Model," Revision O, July 2005. 